\title{
On the nonlinear stability of the triangular points in the circular spatial restricted three-body problem
}

\author{
Daniela Cárcamo-Díaz ${ }^{*}$, Jesús F. Palacián ${ }^{2 * *}$, \\ Claudio Vidal ${ }^{* * *}$, and Patricia Yanguas ${ }^{2^{* * * *}}$ \\ ${ }^{1}$ Universidad del Bío-Bío \\ Grupo de Investigación en Sistemas Dinámicos y Aplicaciones-GISDA, Departamento de Matemática, \\ Facultad de Ciencias, Concepción, VIII Región, Chile \\ ${ }^{2}$ Universidad Pública de Navarra \\ Departamento de Estadística, Informática y Matemáticas and Institute for Advanced Materials, Campus de \\ Arrosadia, 31006 Pamplona, Spain \\ Received August 9, 2019; revised November 29, 2019; accepted January 25, 2020
}

\begin{abstract}
The well-known problem of the nonlinear stability of $L_{4}$ and $L_{5}$ in the circular spatial restricted three-body problem is revisited. Some new results under the light of the concept of Lie (formal) stability are presented. In particular, we provide stability and asymptotic estimates for three specific values of the mass ratio that remained uncovered. Moreover, in many cases we improve the estimates found in the literature.
\end{abstract}

MSC2010 numbers: 37J25, 37N05, 70F15, 70K28

DOI: $10.0000 /$ S1560354700000012

Keywords: restricted three-body problem, $L_{4}$ and $L_{5}$, elliptic equilibria, resonances, formal and Lie stability, exponential estimates

\section{INTRODUCTION}

The nonlinear stability analysis of the points $L_{4}$ and $L_{5}$ is completely solved in the planar case. The spatial case has also been widely studied, mostly first by the Russian school [19, 21, 22, 28] and more recently by the Italian current $[3,8,14,27]$. There is a vast literature on the subject and we do not intend to give all the existing references, but only the most relevant from the point of view of our study. Despite the popularity of the topic, there are still some open questions and part of the existing results can be sharpened.

We will look at this system from the point of view of Lie stability, that is a kind of formal stability. It was Khazin [16] who introduced the concept of Lie stability, although he named it Birkhoff stability in the case of elliptic equilibria. In [11], dos Santos et al. started calling it Lie stability. We will use a powerful criterion given in [7] that enlarges previous criteria on Lie stability and allows us, not only to recover all the formally stable cases reported in previous references, but also to decide on the formal stability of the system for values of the mass parameter that remained pending up to now.

The bound estimates of the solutions over exponentially long times in the Lie stable cases are obtained through a theorem based in the determination of error bounds for adiabatic invariants in Hamiltonian systems [9]. The theorem appears in [7], see also [6]. In many cases this result allows us to get better estimates than the existing ones and even to achieve bounds for the three aforementioned values of the mass ratio. We will make it more precise in sections 4 and 5 .

\footnotetext{
*E-mail: danielacarcamodiaz@gmail.com

${ }^{* *}$ E-mail: palacian@unavarra.es

${ }^{* * *}$ E-mail: clvidal@ubiobio.cl

**** E-mail: yanguas@unavarra.es
} 
The paper is structured as follows. In section 1 we establish the equations of motion of the system. Section 2 is devoted to recall the concepts and the central theorems to establish Lie stability and estimates on the solution. The main results of the manuscript are contained in sections 3 and 4 , the first one including our analysis on the Lie stability of $L_{4}$ and $L_{5}$. Section 4 presents the asymptotic estimates on the solutions in the Lie stable cases. In section 5 we compare our results with the ones existing in the literature and outline our main contributions. In section 6 we establish the existence of invariant 3 -tori encasing $L_{4}$ and $L_{5}$. At the end of the paper there is an appendix containing some useful tables.

The main contributions of our approach are two. First, Theorem 3, where we establish the Lie stability of $L_{4}$ and $L_{5}$ in terms of the mass parameter $\mu$. Second, Theorem 4 , together with Corollary 1 , where the asymptotic bounds for the Lie stable cases are provided. Furthermore, the analysis performed in section 5 makes clear that our estimates enhance those obtained in [3] and other references excepting a few situations, namely: (a) when the frequency vector associated to the formal first integrals defined in (2.1) is not Diophantine and (b) when the parameter $\mu$ lies in the interval $\left(\mu_{1}, \mu_{2}\right)$, with $\mu_{i}$ given in Notation 2 and Notation 3 , and such that the corresponding frequency vector given in Definition 1 is a Pythagorean triple, see Definition 2. Details on the comparison appear in section 5. Finally, the cases not considered in [3], namely the values $\mu_{3}$, $\mu_{(3,3,-2)}$ and $\mu_{(0,3,1)}$, have been proved to be Lie stable, see section 3 . Their asymptotic estimates are given in Corollary 1 . The case $\mu_{(1,3,0)}$, also not treated in [3], is unstable, as it is proved in section 3 .

\section{SETTING OF THE PROBLEM}

We consider the motion in the three-dimensional space of an infinitesimal particle under the gravitational attraction of two bodies with masses $m_{1}$ and $m_{2}$ that describe circular orbits around their common centre of mass (see for example [22] or [29] for details). The Hamilton function associated to this system in rectangular coordinates $(x, y, z, X, Y, Z)$ in a rotating reference frame is:

$$
\mathcal{H}=\frac{1}{2}\left(X^{2}+Y^{2}+Z^{2}\right)-(x Y-X y)-\frac{\mu}{\sqrt{(x+\mu-1)^{2}+y^{2}+z^{2}}}-\frac{1-\mu}{\sqrt{(\mu+x)^{2}+y^{2}+z^{2}}} .
$$

It represents an autonomous system with three degrees of freedom depending on the parameter $\mu$, that stands for the quotient $m_{2} /\left(m_{1}+m_{2}\right)$. Assuming $m_{1} \geq m_{2}$, then $\mu \in(0,1 / 2]$. The masses $m_{1}$ and $m_{2}$ are located at the points $(-\mu, 0,0)$ and $(1-\mu, 0,0)$, respectively, in the coordinate space. The Hamiltonian system has five equilibria, the Euler points $L_{1}, L_{2}$ and $L_{3}$, which are unstable for all $\mu$, and the Lagrangian (also called triangular) points $L_{4}$ and $L_{5}$, whose stability depends on the parameter $\mu$. The coordinates of the Lagrangian equilibrium points $L_{4}$ and $L_{5}$ in the sixdimensional phase space are $(1 / 2-\mu, \pm \sqrt{3} / 2,0, \mp \sqrt{3} / 2,1 / 2-\mu, 0)$, where the upper sign applies for $L_{4}$ and the lower sign does for $L_{5}$. The stability of both equilibria is the same, so from now on we only refer to the point $L_{4}$, although the same conclusions are valid for $L_{5}$.

We translate the equilibrium solution $L_{4}$ to the origin by means of the linear change of coordinates given by $x=x_{1}+1 / 2-\mu, y=y_{1}+\sqrt{3} / 2, z=z_{1}, X=X_{1}-\sqrt{3} / 2, Y=Y_{1}+1 / 2-$ $\mu, Z=Z_{1}$. Then, Hamiltonian function (1.1) is expanded in Taylor series around $\mathbf{0}$, constant terms are dropped and we get a Hamiltonian of the form

$$
H=H_{2}+H_{3}+\cdots+H_{j}+\cdots,
$$

where

$$
\begin{aligned}
H_{2}= & \frac{1}{8}\left(x_{1}^{2}-5 y_{1}^{2}+4 z_{1}^{2}\right)+\frac{1}{2}\left(X_{1}^{2}+Y_{1}^{2}+Z_{1}^{2}\right)-\left(x_{1} Y_{1}-X_{1} y_{1}\right)-\frac{3}{4} \sqrt{3} x_{1} y_{1}(1-2 \mu), \\
H_{3}= & \frac{1}{16}\left[3 \sqrt{3} y_{1}\left(x_{1}^{2}+y_{1}^{2}-4 z_{1}^{2}\right)-x_{1}\left(7 x_{1}^{2}-33 y_{1}^{2}+12 z_{1}^{2}\right)(1-2 \mu)\right], \\
H_{4}= & \frac{1}{128}\left(37 x_{1}^{4}-3 y_{1}^{4}-48 z_{1}^{4}\right)+\frac{3}{64}\left(-41 x_{1}^{2} y_{1}^{2}+4 x_{1}^{2} z_{1}^{2}+44 y_{1}^{2} z_{1}^{2}\right) \\
& +\frac{5}{32} \sqrt{3} x_{1} y_{1}\left(5 x_{1}^{2}-9 y_{1}^{2}+12 z_{1}^{2}\right)(1-2 \mu) .
\end{aligned}
$$


The linearisation matrix associated to $L_{4}$ is

$$
B=\left(\begin{array}{cccccc}
0 & 1 & 0 & 1 & 0 & 0 \\
-1 & 0 & 0 & 0 & 1 & 0 \\
0 & 0 & 0 & 0 & 0 & 1 \\
-\frac{1}{4} & \frac{3}{4} \sqrt{3}(1-2 \mu) & 0 & 0 & 1 & 0 \\
\frac{3}{4} \sqrt{3}(1-2 \mu) & \frac{5}{4} & 0 & -1 & 0 & 0 \\
0 & 0 & -1 & 0 & 0 & 0
\end{array}\right) .
$$

The eigenvalues of matrix $B$ read $\pm \lambda_{1}, \pm \lambda_{2}, \pm \lambda_{3}$, and are pure imaginary whenever $0<\mu<\mu_{R}=$ $\frac{1}{2}(1-\sqrt{69} / 9)$ with $\lambda_{1}=i \omega_{1}, \lambda_{2}=i \omega_{2}, \lambda_{3}=i \omega_{3}$ and

$$
\omega_{1}=\frac{\sqrt{1+\sqrt{1-27 \mu+27 \mu^{2}}}}{\sqrt{2}}, \omega_{2}=\frac{\sqrt{1-\sqrt{1-27 \mu+27 \mu^{2}}}}{\sqrt{2}}, \omega_{3}=1 .
$$

The value $\mu_{R}$ is the so-called Gascheau's or Routh's critical value. Note that when $0<\mu<\mu_{R}$ one has

$$
0<\omega_{2}<\frac{\sqrt{2}}{2}<\omega_{1}<1 \text { and } \omega_{1}^{2}+\omega_{2}^{2}=1
$$

It is also convenient to express $\mu$ in terms of $\omega_{1}, \omega_{2}$, leading to

$$
\mu=\frac{1}{2}\left(1-\frac{\sqrt{27-16 \omega_{1}^{2}+16 \omega_{1}^{4}}}{3 \sqrt{3}}\right)=\frac{1}{2}\left(1-\frac{\sqrt{27-16 \omega_{2}^{2}+16 \omega_{2}^{4}}}{3 \sqrt{3}}\right) .
$$

Observe that, taking into account (1.3), $\omega_{1}=\sqrt{2} / 2$ corresponds to $\mu=\mu_{R}$ while $\omega_{1}=1$ is associated to $\mu=0$. When $\mu>\mu_{R}$ the equilibrium $L_{4}$ is of focus-centre type, therefore unstable as it comes from a symplectic system and the eigenvalues are $\pm \lambda \pm i \nu$ with $\lambda, \nu$ positive numbers. When $\mu=\mu_{R}$ the linear system is not diagonalisable, thus the equilibrium points are not of elliptic nature. This case was proved to be Liapunov stable in the planar case in $[17,24]$ and formally stable in the spatial case, see [22]. In the context of the planar restricted problem with $\mu=\mu_{R}$ it is also worth mentioning papers [28], where a first attempt to study nonlinear stability in the sense of Liapunov is performed, and [2], where the author shows that, in a neighbourhood of $\mu_{R}$, for most initial conditions, trajectories are conditionally periodic. An account of the stability achievements when $\mu \in\left(0, \mu_{R}\right]$, also for the planar case, appears in [23]. In our analysis we focus on the interval $\left(0, \mu_{R}\right)$ for $\mu$, equivalently $(\sqrt{2} / 2,1)$ for $\omega_{1}$ and $(0, \sqrt{2} / 2)$ for $\omega_{2}$.

There are several ways of performing the normal form transformation of Hamiltonian $H$ in (1.2). The most standard one consists in introducing a real linear symplectic change of coordinates to put $\mathrm{H}_{2}$ in linear normal form by using the eigenvalues and eigenvectors of matrix $B$. We call the transformed variables $\mathbf{x}=\left(q_{1}, q_{2}, q_{3}, p_{1}, p_{2}, p_{3}\right)$, where the $q_{i}$ stand for coordinates and $p_{i}$ do for their conjugate momenta. Then, a complex linear change is introduced to express $\mathrm{H}_{2}$ in complex diagonal form. Next, the two changes are applied to the higher-order terms. Finally, a procedure based on Lie transformations is applied to normalise the terms from $H_{3}$ on so that the resulting Hamiltonian at each step commutes with $\mathrm{H}_{2}$. This process is executed up to a finite order and in most of the cases in this problem order two is enough, which means, including the polynomials of degree four that define $H_{4}$.

In general we denote by $\mathcal{H}^{p}$ the normal form truncated at order $p-2$, in other words, at degree $p$ in terms of rectangular coordinates, thus

$$
\mathcal{H}^{p}=H_{2}+\mathcal{H}_{3}+\cdots+\mathcal{H}_{p}
$$

and the Poisson brackets $\left\{H_{2}, \mathcal{H}_{k}\right\}=0$ for $k=2, \ldots, p$. In the setting of elliptic equilibria the normal form is the so-called Birkhoff normal form, see for instance Theorem 5.5 in [1]. 
To achieve the normal form we need to calculate the associated generating function that is used to define the symplectic transformation. Since the expressions of the two terms of the generating transformation are lengthy, we do not include them here, but they are available upon request from the authors. The calculations have been performed in terms of the parameter $\omega_{1}$ using symbolic arithmetic.

Now, we introduce the usual action-angle coordinates, say

$$
(\mathbf{I}, \theta)=\left(I_{1}, I_{2}, I_{3}, \theta_{1}, \theta_{2}, \theta_{3}\right)
$$

where $I_{j}=\frac{1}{2}\left(q_{j}^{2}+p_{j}^{2}\right)$ are the actions conjugate to the angles $\theta_{j}=\tan ^{-1}\left(p_{j} / q_{j}\right)$ with $j=1,2,3$. Then $\mathrm{H}_{2}$ is converted into

$$
H_{2}=\omega_{1} I_{1}-\omega_{2} I_{2}+\omega_{3} I_{3} .
$$

Notice that $H_{2}$ is indefinite when $\mu \in\left(0, \mu_{R}\right)$.

The normalised Hamiltonian in action-angle coordinates reads as

$$
H=H_{2}+\mathcal{H}_{4}+\cdots,
$$

where

$$
\mathcal{H}_{4}=c_{200} I_{1}^{2}+c_{110} I_{1} I_{2}+c_{101} I_{1} I_{3}+c_{020} I_{2}^{2}+c_{011} I_{2} I_{3}+c_{002} I_{3}^{2}
$$

and

$$
\begin{aligned}
& c_{200}=\frac{\omega_{2}^{2}\left(124 \omega_{1}^{4}-696 \omega_{1}^{2}+81\right)}{144\left(1-2 \omega_{1}^{2}\right)^{2}\left(1-5 \omega_{1}^{2}\right)}, \quad c_{110}=-\frac{\omega_{1} \omega_{2}\left(64 \omega_{1}^{2} \omega_{2}^{2}+43\right)}{6\left(1-5 \omega_{1}^{2}\right)\left(1-2 \omega_{1}^{2}\right)\left(1-5 \omega_{2}^{2}\right)\left(1-2 \omega_{2}^{2}\right)}, \\
& c_{101}=-\frac{8 \omega_{1} \omega_{2}^{2}}{3\left(1-2 \omega_{1}^{2}\right)\left(4-\omega_{1}^{2}\right)}, \quad c_{020}=\frac{\omega_{1}^{2}\left(124 \omega_{1}^{4}+448 \omega_{1}^{2}-491\right)}{144\left(1-2 \omega_{1}^{2}\right)^{2}\left(1-5 \omega_{2}^{2}\right)}, \\
& c_{011}=\frac{8 \omega_{2} \omega_{1}^{2}}{3\left(1-2 \omega_{2}^{2}\right)\left(4-\omega_{2}^{2}\right)}, \quad \quad c_{002}=-\frac{\omega_{1}^{2} \omega_{2}^{2}}{3\left(4-\omega_{1}^{2}\right)\left(4-\omega_{2}^{2}\right)} .
\end{aligned}
$$

If we discard possible higher-order resonances, odd terms in the normal form Hamiltonian, say $\mathcal{H}_{3}, \mathcal{H}_{5}, \ldots$, are zero. Coefficients $c_{200}, c_{110}, c_{020}$ were already calculated in [10] in the context of the planar case.

Taking into consideration (1.4), there are only two resonant cases where the above normal form does not apply. Specifically,

(i) $\omega_{1}=\frac{2}{\sqrt{5}}, \omega_{2}=\frac{1}{\sqrt{5}}$ thus, $\omega_{1} / \omega_{2}=2$ and

$$
\mu=\mu_{(1,2,0)}=\frac{1}{2}\left(1-\frac{\sqrt{1833}}{45}\right) .
$$

(ii) $\omega_{1}=\frac{3}{\sqrt{10}}, \omega_{2}=\frac{1}{\sqrt{10}}$ thus, $\omega_{1} / \omega_{2}=3$ and

$$
\mu=\mu_{(1,3,0)}=\frac{1}{2}\left(1-\frac{\sqrt{213}}{15}\right) .
$$

For these two sets of values some denominators of the generating function vanish and, as a consequence, the generic normal form Hamiltonian already calculated is not valid. Thus, in these cases we should compute specific normal forms that we shall show below. Notice that, apart from these two cases, $\mathcal{H}^{4}$ does not contain any resonant terms under conditions (1.4). 


\section{ON LIE STABILITY}

In this section we review the main concepts and results related to Lie stability with the aim of applying them in section 3 . We start by recalling the notion of resonance.

Definition 1. The system related with Hamiltonian (1.7) presents a resonance relation if there exists an integer vector $\mathbf{k}=\left(k_{1}, k_{2}, k_{3}\right) \neq \mathbf{0}$ such that

$$
k_{1} \omega_{1}-k_{2} \omega_{2}+k_{3} \omega_{3}=0 .
$$

The 1-norm of vector $\mathbf{k},|\mathbf{k}|_{1}=\left|k_{1}\right|+\left|k_{2}\right|+\left|k_{3}\right|$, is called the order of the resonance, while $\mathbf{k}$ is known as the resonance vector and $\omega=\left(\omega_{1}, \omega_{2}, \omega_{3}\right)$ stands for the frequency vector.

For instance, resonance vectors corresponding to

$$
\left(\omega_{1}, \omega_{2}, \omega_{3}\right)=\left(\frac{2}{\sqrt{5}}, \frac{1}{\sqrt{5}}, 1\right) \text { and }\left(\frac{3}{\sqrt{10}}, \frac{1}{\sqrt{10}}, 1\right),
$$

which are associated to (1.9) and (1.10), are $\left(k_{1}, k_{2}, k_{3}\right)=(1,2,0)$ and $(1,3,0)$, respectively.

Notation 1. The notation $\mu_{\left(k_{1}, k_{2}, k_{3}\right)}$ means taking the value of $\mu \in\left(0, \mu_{R}\right)$ associated to the resonance vector $\mathbf{k}=\left(k_{1}, k_{2}, k_{3}\right)$. In fact, along the paper we will use the same notation for the $\mu_{i}$ and $\mu_{\left(k_{1}, k_{2}, k_{3}\right)}$ as in reference [3].

Definition 2. Consider the frequency vector $\omega=\left(m / n, \sqrt{n^{2}-m^{2}} / n, 1\right)$, with $m, n \in \mathbb{Z}^{+}, 0<m<$ $n$ and $m / n$ irreducible. Vector $\left(m, \sqrt{n^{2}-m^{2}}, n\right)$ is a Pythagorean triple if $n^{2}-m^{2}$ is a perfect square or, equivalently, $\omega_{2} \in \mathbb{Q}$. In this case we say that vector $\omega$ is associated with a Pythagorean triple.

We will notice that the previous concept is crucial to decide on Lie stability.

Definition 3. We say that the origin of $\mathbb{R}^{6}$ in (1.2) is Lie stable if there exists $m>2$ such that the truncated Hamiltonian system in Birkhoff normal form associated to $\mathcal{H}^{j}$ is stable in the sense of Liapunov for any (arbitrary) $j \geq m$.

Definition 4. We say that the origin of $\mathbb{R}^{6}$ in (1.2) is formally stable if there exists a real formal power series $G(\mathbf{x})$, possibly divergent, which is an integral of $H$ in the formal sense, and is positive definite near $\mathbf{x}=\mathbf{0}$.

Remark 1. The generalisation to $n$ degrees of freedom of the definitions of Lie and formal stability is straightforward [11]. Lie stability is a type of formal stability, see for instance [12]. As the normal form transformation is carried out only to a finite order, checking Lie stability for the system in normal form is equivalent to checking Lie stability for any system previous to the normal form calculations.

Statement 1. Suppose $\left\{\mathbf{k}^{1}, \ldots, \mathbf{k}^{s}\right\}$ is a basis of the $\mathbb{Z}$-module $M_{\omega}$ associated to the possible resonances of $H_{2}$, where $0 \leq s \leq 2$. The null space of $M_{\omega}$ is a vector subspace of $\mathbb{R}^{3}$ spanned by the vectors $\left\{\mathbf{a}_{1}, \ldots, \mathbf{a}_{d}\right\}$ with $d=3-s$ that satisfy $\mathbf{a}_{i} \cdot \mathbf{k}^{j}=0$, see details in [11], [12]. By setting $F_{l}=\mathbf{a}_{l} \cdot \mathbf{I}$ with $l=1, \ldots, d$, we get the independent (formal) first integrals of the normalised Hamiltonian (1.7).

Definition 5. We define the set

$$
S=\left\{\mathbf{I} \mid F_{1}(\mathbf{I})=\ldots=F_{d}(\mathbf{I})=0\right\},
$$

which was first introduced in [11].

The set $S$ contains the essential vectors to evaluate the Hamiltonian and decide on the Lie stability of the system. We note that $0 \leq \operatorname{dim} S \leq s$. 
Statement 2. The quadratic part of $H$ in terms of the formal first integrals $F_{k}$ assumes the form

$$
H_{2}(\mathbf{I})=\sum_{k=1}^{d} \sigma_{k} F_{k}(\mathbf{I}),
$$

where $\sigma_{k}$ are linear combinations of $\omega_{j}$, see for instance [13]. The coefficients $\sigma_{k}$ are rationally independent.

Definition 6. It is said that vector $\sigma=\left(\sigma_{1}, \ldots, \sigma_{d}\right)$ satisfies a Diophantine condition when there are fixed constants $c>0$ and $\nu \geq d-1$ such that

$$
\forall \mathbf{k} \in \mathbb{Z}^{d} \backslash\{\mathbf{0}\}, \quad|\mathbf{k} \cdot \sigma| \geq c|\mathbf{k}|_{1}^{-\nu} .
$$

The symbol | | stands for the Euclidean norm.

At this point we recall two results that will be applied in the present paper in order to analyse the nonlinear stability of $L_{4}$ and give time estimates in the stable cases. We start with the theorem on Lie stability, as stated in $[6,7]$, that will be applied in section 3 .

Assume that Hamiltonian (1.1) is expressed as $H$ in (1.2) after sufficient manipulations and also that its normal form, up to an order $p$ high enough, is given in (1.6). Then, the following result applies.

Theorem 1. (A) Suppose there is an integer $j \geq 3$ with $\mathcal{H}^{j}\left(\mathbf{I}, \phi_{1}, \ldots, \phi_{s}\right) \neq 0$ for all $\mathbf{I} \in S \backslash\{\mathbf{0}\}$, $\left\{\phi_{1}, \ldots, \phi_{s}\right\} \in \mathbb{T}^{s}, \phi_{i}=\mathbf{k}_{i} \cdot \theta$ such that for all $i$ with $3 \leq i<j, \mathcal{H}^{i}\left(\mathbf{I}, \phi_{1}, \ldots, \phi_{s}\right)$ does not change sign for $\mathbf{I} \in S \backslash\{\mathbf{0}\},\left\{\phi_{1}, \ldots, \phi_{s}\right\} \in \mathbb{T}^{s}$, where $|\mathbf{I}|$ is small enough. Then, the origin of $\mathbb{R}^{6}$ is Lie stable for the Hamiltonian system (1.2).

(B) Suppose there is an integer $i \geq 3$ such that $\mathcal{H}^{i}\left(\mathbf{I}, \phi_{1}, \ldots, \phi_{s}\right)$ changes sign for some $\mathbf{I} \in$ $S \backslash\{\mathbf{0}\},\left\{\phi_{1}, \ldots, \phi_{s}\right\} \in \mathbb{T}^{s}$, where $|\mathbf{I}|$ is small enough. Then, there is no index $j>i$ such that $\mathcal{H}^{j}\left(\mathbf{I}, \phi_{1}, \ldots, \phi_{s}\right) \neq 0$ for $\mathbf{I} \in S \backslash\{\mathbf{0}\},\left\{\phi_{1}, \ldots, \phi_{s}\right\} \in \mathbb{T}^{s}$ with $|\mathbf{I}|$ sufficiently small.

According to the previous theorem, the Lie stability analysis consists in calculating the set $S$ introduced above, which is a linear subspace of $\mathbb{R}^{6}$ that is contained into the orthogonal space related to the frequency vector $\omega$. The Hamiltonian in normal form is computed only up to a suitable order, checking whether its truncation vanishes only at the origin of $S$. When it so happens, then Lie stability is obtained.

Remark 2. When $S=\{\mathbf{0}\}$ there is always Lie stability, see details in [12], [7].

For the Lie stable equilibria we will give an estimate of the solution's evolution according to the following theorem $[6,7]$. Assume that $\mathbf{x}\left(t, \mathbf{x}_{0}\right)$ is a solution of the Hamiltonian system associated to $H$ in (1.2) with initial condition $\mathbf{x}_{0}$. The following result holds.

Theorem 2. If the real analytic Hamiltonian (1.2) has the origin of $\mathbb{R}^{6}$ as a formally stable equilibrium according to hypotheses (A) of Theorem 1, while the frequency vector $\sigma$ satisfies the Diophantine condition (2.2), then there exist $C>0, E>0, a>1$ and $\varepsilon_{0}>0$ such that for all $\varepsilon \in\left(0, \varepsilon_{0}\right)$, and for all $\mathbf{x}_{0}$ with $\left|\mathbf{x}_{0}\right|<\varepsilon$ we have

$$
\left|\mathbf{x}\left(t, \mathbf{x}_{0}\right)\right|<a \varepsilon^{2 / j} \quad \text { for all } t \text { with } \quad 0 \leq t \leq T=C \exp \left(\frac{E}{\varepsilon^{1 /(\nu+1)}}\right) .
$$

The proofs of theorems 1 and 2 appear in $[6,7]$.

\section{LIE STABILITY OF $L_{4}$}

Let us start by analysing the cases where the normal form (1.8) does not apply. Recall from section 1 that (1.8) is not valid valid for $\omega_{1}=2 / \sqrt{5}$, i.e. $\mu=\mu_{(1,2,0)}$, nor for $\omega_{1}=3 / \sqrt{10}$, that is, $\mu=\mu_{(1,3,0)}$. A specific normal form is calculated for each of these values concluding instability in both cases, as it is also inferred from the planar problem [19, 20]. 
Case $\mu_{(1,2,0)}$ : For $\omega_{1}=2 / \sqrt{5}$ the normal form up to degree 3 is

$$
\mathcal{H}^{3}\left(\mathbf{I}, \phi_{1}\right)=\frac{1}{\sqrt{5}}\left(2 I_{1}-I_{2}\right)+I_{3}+\frac{1}{5^{3 / 4} \cdot 3312 \sqrt{109}} \sqrt{I_{1}} I_{2}\left(3401 \sqrt{5} \cos \phi_{1}-3155 \sqrt{611} \sin \phi_{1}\right),
$$

where $\phi_{1}=\theta_{1}+2 \theta_{2}$. Notice that $S=\left\{\left(I_{1}, 2 I_{1}, 0\right) \mid I_{1} \geq 0\right\}$. Then, we take $\mathbf{I}$ in $S$ and get

$$
\mathcal{H}^{3}\left(\mathbf{I}, \phi_{1}\right)=\frac{1}{5^{3 / 4} \cdot 1656 \sqrt{109}} I_{1}^{3 / 2}\left(3401 \sqrt{5} \cos \phi_{1}-3155 \sqrt{611} \sin \phi_{1}\right) .
$$

As the coefficient of $I_{1}^{3 / 2}$ has a simple zero then, by Theorem 3.1 in [11], the equilibrium is unstable.

Case $\mu_{(1,3,0)}$ : For $\omega_{1}=3 / \sqrt{10}$ the normal form up to terms of degree four is

$$
\begin{aligned}
\mathcal{H}^{4}\left(\mathbf{I}, \phi_{1}\right)= & \frac{1}{\sqrt{10}}\left(3 I_{1}-I_{2}\right)+I_{3}+\frac{309}{2240} I_{1}^{2}+\frac{79}{320} I_{2}^{2}-\frac{1}{403} I_{3}^{2}-\frac{1219}{560} I_{1} I_{2}+\frac{\sqrt{10}}{31} I_{1} I_{3}+\frac{\sqrt{10}}{13} I_{2} I_{3} \\
& -\frac{1}{32560 \sqrt{28083}} \sqrt{I_{1}} I_{2}^{3 / 2}\left(24146471 \cos \phi_{1}+143827 \sqrt{710} \sin \phi_{1}\right),
\end{aligned}
$$

with $\phi_{1}=\theta_{1}+3 \theta_{2}$. On this occasion $S=\left\{\left(I_{1}, 3 I_{1}, 0\right) \mid I_{1} \geq 0\right\}$. Evaluating $\mathcal{H}^{4}$ in $S$ we get

$$
\mathcal{H}^{4}\left(\mathbf{I}, \phi_{1}\right)=\frac{-3}{4267118240} I_{1}^{2}\left(5932056339+338050594 \sqrt{9361} \cos \phi_{1}+2013578 \sqrt{6646310} \sin \phi_{1}\right) .
$$

Then, the coefficient of $I_{1}^{2}$ in $\mathcal{H}^{4}\left(\mathbf{I}, \phi_{1}\right)$ has a simple zero and then, by Theorem 3.1 in [11], we achieve instability.

Now we study the rest of the cases starting with the determination of the set $S$. This passes through the construction of the formal integrals $F_{i}$ associated to $H_{2}$. We can have one, two or three linearly independent integrals. The basic relations to be taken into account are written as

$$
\omega_{1} I_{1}-\omega_{2} I_{2}+I_{3}=0, \quad k_{1} \omega_{1}-k_{2} \omega_{2}+k_{3}=0,
$$

with $k_{1}, k_{2}, k_{3} \in \mathbb{Z}$ and $I_{1}, I_{2}, I_{3} \geq 0$. The following situations are in order:

$\left(a_{1}\right)$ If $\omega_{1}, \omega_{2} \in \mathbb{Q}$, then the frequency vector is associated to a Pythagorean triple. We get $F_{1}=\omega_{1} I_{1}-\omega_{2} I_{2}+I_{3}, d=1, s=2$ and

$$
S=\left\{\left(I_{1}, \frac{1}{\omega_{2}}\left(\omega_{1} I_{1}+I_{3}\right), I_{3}\right) \mid I_{1}, I_{3} \geq 0\right\} .
$$

Considering $\mathbf{I} \in S \backslash\{\mathbf{0}\}$, taking into account (1.4) to express $\omega_{2}$ as a function of $\omega_{1}$, and replacing everything in (1.8) we arrive at

$$
\mathcal{H}^{4}(\mathbf{I})=\beta_{1} I_{1}^{2}+\beta_{2} I_{1} I_{3}+\beta_{3} I_{3}^{2},
$$

with

$$
\begin{aligned}
& \beta_{1}=\frac{644 \omega_{1}^{8}-1288 \omega_{1}^{6}+1185 \omega_{1}^{4}-541 \omega_{1}^{2}+36}{16\left(1-\omega_{1}^{2}\right)\left(1-2 \omega_{1}^{2}\right)^{2}\left(1-5 \omega_{1}^{2}\right)\left(4-5 \omega_{1}^{2}\right)}, \\
& \beta_{2}=\frac{\omega_{1}\left(18580 \omega_{1}^{12}-67928 \omega_{1}^{10}+70827 \omega_{1}^{8}+30890 \omega_{1}^{6}-62113 \omega_{1}^{4}+22128 \omega_{1}^{2}-8496\right)}{72\left(1-\omega_{1}^{2}\right)\left(1-2 \omega_{1}^{2}\right)^{2}\left(1-5 \omega_{1}^{2}\right)\left(3+\omega_{1}^{2}\right)\left(4-\omega_{1}^{2}\right)\left(4-5 \omega_{1}^{2}\right)}, \\
& \beta_{3}=\frac{\omega_{1}^{2}\left(960 \omega_{1}^{10}-7364 \omega_{1}^{8}+29940 \omega_{1}^{6}-48219 \omega_{1}^{4}+24155 \omega_{1}^{2}-444\right)}{144\left(1-\omega_{1}^{2}\right)\left(1-2 \omega_{1}^{2}\right)^{2}\left(3+\omega_{1}^{2}\right)\left(4-\omega_{1}^{2}\right)\left(4-5 \omega_{1}^{2}\right)} .
\end{aligned}
$$

We have to determine when there is no sign-change in $\mathcal{H}^{4}(\mathbf{I})$, keeping in mind that $I_{1}, I_{3}$ should be non-negative. First, notice that there is only one solution of $\beta_{1}=0$ in the interval $(1 / \sqrt{2}, 1)$. 
Notation 2. We denote

$$
\omega_{1}^{*}=\frac{1}{2} \sqrt{2+\sqrt{\frac{2}{161}(-219+\sqrt{199945})}} \approx 0.959622914235418
$$

the only solution of $\beta_{1}=0$ in the interval $(1 / \sqrt{2}, 1)$. From (1.5) the corresponding value of $\mu$ is

$$
\mu_{1}=\frac{1}{2}\left(1-\frac{1}{3} \sqrt{\frac{1}{483}(3265+2 \sqrt{199945})}\right),
$$

following the notation in [3].

Second, notice that $\beta_{3}=0$ has only one solution in the interval $(1 / \sqrt{2}, 1)$.

Notation 3. We denote $\omega_{1}^{\sharp}$ the only solution of $\beta_{3}=0$ in the interval $(1 / \sqrt{2}, 1)$. It is the square root of a root of the fifth-degree polynomial $960 x^{5}-7364 x^{4}+29940 x^{3}-48219 x^{2}+$ $24155 x-444$ lying in the interval $(1 / \sqrt{2}, 1)$. An approximation of it is

$$
\omega_{1}^{\sharp} \approx 0.935871439168618 .
$$

From (1.5) the corresponding value of $\mu$ is

$$
\mu_{2} \approx 0.016376755355816
$$

following the notation in reference [3].

Using Mathematica version 12, in particular applying the specific routines of solving equations and inequalities and eliminating quantifiers, we have proved that $\mathcal{H}^{4}$ keeps the same sign for all $\omega_{1} \in D \cap \mathbb{Q}$, where

$$
D=\left(\frac{1}{\sqrt{2}}, 1\right) \backslash\left(\left\{\frac{2}{\sqrt{5}}\right\} \cup\left[\omega_{1}^{\sharp}, \omega_{1}^{*}\right]\right) .
$$

It is stressed that $\mathcal{H}^{4}(\mathbf{I})$ with $\mathbf{I} \in S \backslash\{\mathbf{0}\}$ does not change sign for $\omega_{1} \in D$ regardless of whether $\omega_{1}$ is rational or not.

At this point it is rather convenient to introduce the rational root test [25]: Given a polynomial in the variable $x$ of degree $n$, say, $r(x)=a_{n} x^{n}+a_{n-1} x^{n-1}+\cdots+a_{1} x^{1}+a_{0}=0$, where $a_{0}$, $a_{1}, \ldots, a_{n}$ are integers, the rational root test says that for the polynomial $r(x)$ to have a rational solution of the form $p / q$ (irreducible fraction), $q$ must divide $a_{n}$ and $p$ must divide $a_{0}$.

Applying the criterion given above we have proved that $\omega_{1}^{\sharp}, \omega_{2}^{\sharp}=\left(1-\omega_{1}^{\sharp 2}\right)^{1 / 2}$ belong to $\mathbb{R} \backslash \mathbb{Q}$. Moreover, we have checked that there is no integer vector $\mathbf{k} \neq \mathbf{0}$ such that $\mathbf{k} \cdot\left(\omega_{1}^{\sharp},-\omega_{2}^{\sharp}, 1\right)=0$. So this case will be tackled in $\left(b_{1}\right)$.

Regarding $\omega_{1}^{*}$ we have also checked that it is irrational and so is the corresponding $\omega_{2}^{*}=\left(1-\omega_{1}^{* 2}\right)^{1 / 2}$. Additionally there is no integer vector $\mathbf{k} \neq \mathbf{0}$ such that $\mathbf{k} \cdot\left(\omega_{1}^{*},-\omega_{2}^{*}, 1\right)=0$. Therefore, this case has to be analysed in $\left(b_{1}\right)$ too.

Thence, by virtue of Theorem 1 the equilibrium $L_{4}$ is Lie stable for $\omega_{1} \in D \cap \mathbb{Q}$ and $\omega_{2} \in \mathbb{Q}$ related to a Pythagorean triple.

An example lying in this class is for instance $\omega_{1}=4 / 5, \omega_{2}=3 / 5$, which yields Lie stability. However, choosing $\omega_{1}=35 / 37, \omega_{2}=12 / 37$, as $\omega_{1} \in\left[\omega_{1}^{\sharp}, \omega_{1}^{*}\right]$, one has that $\mathcal{H}^{4}$ changes sign in $S$ and then we cannot decide on its stability. This corresponds to a Pythagorean triple and it is associated to a resonance of order eight, say $\left(k_{1}, k_{2}, k_{3}\right)=(1,6,1)$. It is indeed the lowest order for a resonance corresponding to a Pythagorean triple in the interval $\left(\omega_{1}^{\sharp}, \omega_{1}^{*}\right)$. 
$\left(a_{2}\right)$ If $\omega_{1} \in \mathbb{Q}$ and $\omega_{2} \in \mathbb{R} \backslash \mathbb{Q}$, then the frequency vector is not associated to a Pythagorean triple, and one gets $F_{1}=\omega_{1} I_{1}+I_{3}, F_{2}=I_{2}$. Thus $d=2, s=1$ and

$$
S=\left\{\left(I_{1}, I_{2}, I_{3}\right) \mid \omega_{1} I_{1}+I_{3}=0, I_{2}=0, I_{1}, I_{3} \geq 0\right\}=\{\mathbf{0}\} .
$$

Then, by applying Theorem 1 we conclude that $L_{4}$ is Lie stable.

An example in $\left(a_{2}\right)$ is $\omega_{1}=6 / 7$ and $\omega_{2}=\sqrt{13} / 7$.

$\left(b_{1}\right)$ If $\omega_{1}, \omega_{2} \in \mathbb{R} \backslash \mathbb{Q}$ and there is no integer vector $\mathbf{k}=\left(k_{1}, k_{2}, k_{3}\right) \neq(0,0,0)$ such that $\mathbf{k} \cdot$ $\left(\omega_{1},-\omega_{2}, 1\right)=0$ then there are no resonances among the main frequencies. Hence, $F_{j}=I_{j}$ for $j=1,2,3, d=3, s=0$ and $S=\{\mathbf{0}\}$. So Lie stability holds.

As we have seen in $\left(a_{1}\right)$, the values $\omega_{1}^{\sharp}, \omega_{1}^{*}$, correspondingly $\mu_{2}, \mu_{1}$, belong to this case, so both of them lead to Lie stability.

Two more examples are $\omega_{1}=6 / \sqrt{41}, \omega_{2}=\sqrt{5 / 41}$ and $\omega_{1}=2 / e, \omega_{2}=\sqrt{e^{2}-4} / e$.

$\left(b_{2}\right)$ If $\omega_{1}, \omega_{2} \in \mathbb{R} \backslash \mathbb{Q}$ and there is an integer vector $\mathbf{k}=\left(k_{1}, k_{2}, k_{3}\right) \neq(0,0,0)$ such that $\mathbf{k}$. $\left(\omega_{1},-\omega_{2}, 1\right)=0$, then

$$
\omega_{1}=\frac{k_{2}}{k_{1}} \omega_{2}-\frac{k_{3}}{k_{1}},
$$

with $k_{1} \neq 0$. Notice that $k_{1}=0$ would imply $-\omega_{2} k_{2}+k_{3}=0$ and then, either $k_{2}=0$, in which case $k_{1}=k_{2}=k_{3}=0$, that is impossible, or $\omega_{2}=k_{3} / k_{2} \in \mathbb{Q}$, contradicting the hypotheses of $\left(b_{2}\right)$. Analogously $k_{2} \neq 0$ because $k_{2}=0$ would lead to $\omega_{1}=-k_{3} / k_{1} \in \mathbb{Q}$, that is not feasible. However, $k_{3}=0$ is possible and this implies $k_{1} \omega_{1}=k_{2} \omega_{2}$. We have to exclude the particular value $\omega_{1}=3 / \sqrt{10}$, as it leads to instability.

Using (3.4) we get

$$
H_{2}=\left(\frac{k_{2}}{k_{1}} I_{1}-I_{2}\right) \omega_{2}-\frac{k_{3}}{k_{1}} I_{1}+I_{3},
$$

from where we deduce that $F_{1}=k_{2} I_{1} / k_{1}-I_{2}, F_{2}=-k_{3} I_{1} / k_{1}+I_{3}, d=2$ and $s=1$.

Consider the set

$$
K=\left\{\left(k_{1}, k_{2}, k_{3}\right) \in \mathbb{Z}^{3} \mid k_{1} \neq 0, k_{2} / k_{1}>0, k_{3} / k_{1} \geq 0\right\} .
$$

If $\mathbf{k} \in K$ then one has $\operatorname{dim} S=1$ with

$$
S=\left\{\left(I_{1}, \frac{k_{2}}{k_{1}} I_{1}, \frac{k_{3}}{k_{1}} I_{1}\right) \mid I_{1} \geq 0\right\} .
$$

Taking $\mathbf{I} \in S$ and using (1.4) and (3.4) we get

$$
\mathcal{H}^{4}(\mathbf{I})=\left(\beta_{1}+\beta_{2} \frac{k_{3}}{k_{1}}+\beta_{3} \frac{k_{3}^{2}}{k_{1}^{2}}\right) I_{1}^{2},
$$

where $\beta_{1}, \beta_{2}$ and $\beta_{3}$ are given in (3.2). In fact $\mathcal{H}^{4}(\mathbf{I})$ can be obtained by replacing $I_{3}=k_{3} I_{1} / k_{1}$ in (3.1). Due to the form acquired by $\mathcal{H}^{4}$, we notice this is a particular situation of $\mathcal{H}^{4}$ in $\left(a_{1}\right)$ and we can conclude that $\mathcal{H}^{4}(\mathbf{I})$ does not change sign in $S \backslash\{\mathbf{0}\}$ when $\omega_{1} \in D \cap(\mathbb{R} \backslash \mathbb{Q})$ with $D$ given in $(3.3)$ and $\omega_{2} \in \mathbb{R} \backslash \mathbb{Q}$. Therefore, Lie stability is accomplished when $\mathbf{k} \in K$.

As in $\left(a_{1}\right)$, we have verified with Mathematica that in the interval $\left[\omega_{1}^{\sharp}, \omega_{1}^{*}\right]$ there is no $\mathbf{k} \in K$ such that $\mathcal{H}^{4}(\mathbf{I})$ vanishes for $\mathbf{I}$ in $S \backslash\{\mathbf{0}\}$, so for the study of this interval we go back to $\left(b_{1}\right)$, concluding Lie stability.

Finally when $\mathbf{k} \notin K$ then, from the first integrals $F_{1}, F_{2}$ given a few lines above one deduces that $S=\{\mathbf{0}\}$, concluding Lie stability. 
An example of the previous situation is $\mu_{(3,3,-2)}$ that will be mentioned in the forthcoming sections. In this case

$$
\omega_{1}=\frac{1}{6}(2+\sqrt{14}), \quad \omega_{2}=\frac{1}{6}(-2+\sqrt{14}),
$$

which are irrational although resonant, the resonance vector being $\mathbf{k}=(3,3,-2) \notin K$. Then, Lie stability holds.

$\left(b_{3}\right)$ If $\omega_{1} \in \mathbb{R} \backslash \mathbb{Q}$ and $\omega_{2} \in \mathbb{Q}$, then we take $F_{1}=I_{1}, F_{2}=-\omega_{2} I_{2}+I_{3}$, from where we get $d=2$, $s=1$ and

$$
S=\left\{\left(0, \frac{1}{\omega_{2}} I_{3}, I_{3}\right) \mid I_{3} \geq 0\right\}
$$

We consider $\mathbf{I} \in S \backslash\{\mathbf{0}\}$, write $\omega_{2}=\left(1-\omega_{1}^{2}\right)^{1 / 2}$ and replace it in (1.8), ending up with $\mathcal{H}^{4}(\mathbf{I})=\beta_{3} I_{3}^{2}$, with $\beta_{3}$ given in (3.2). Notice that we arrive at the same expression replacing $I_{1}=0$ in (3.1). The domain where the normal form is properly defined and where $\beta_{3} \neq 0$ is $(1 / \sqrt{2}, 1) \backslash\left\{2 / \sqrt{5}, \omega_{1}^{\sharp}, 3 / \sqrt{10}\right\}$. Since the values of $\omega_{1}$ that we are discarding are in correspondence with irrational values of $\omega_{2}$, we do not care about them. Therefore, applying Theorem 1 , the equilibrium $L_{4}$ is Lie stable whenever $\omega_{1} \in(1 / \sqrt{2}, 1)$ with $\omega_{1} \in \mathbb{R} \backslash \mathbb{Q}$ and $\omega_{2} \in \mathbb{Q}$.

An example borrowed from reference $[3]$ is $\mu_{(0,3,1)}$. On this occasion $\omega_{1}=2 \sqrt{2} / 3 \in \mathbb{R} \backslash \mathbb{Q}$, $\omega_{2}=1 / 3 \in \mathbb{Q}$, concluding Lie stability.

In Table 1 we have put the resonant cases corresponding to $\operatorname{dim} S=0$ until the eighth-order of resonance whereas in Table 2 we have written the resonant cases corresponding to $\operatorname{dim} S=1$ also to order 8. Note that $\sigma$ is always a Diophantine vector. Both tables appear in the Appendix.

Taking into account Notation 2, Notation 3 and $\mu_{(1,2,0)}, \mu_{(1,3,0)}$ introduced respectively in (1.9) and (1.10), we summarise our main result on the Lie stability of $L_{4}$ in the spatial case of the restricted circular three body problem as follows.

Theorem 3. For $0<\mu<\mu_{R}$ the equilibrium point $L_{4}$ is Lie stable for the Hamiltonian system related to (1.1), excepting the unstable situations $\mu_{(1,2,0)}, \mu_{(1,3,0)}$ and the values $\mu \in\left(\mu_{1}, \mu_{2}\right)$ leading to a Pythagorean triple.

\section{ASYMPTOTIC ESTIMATES}

In this section we apply Theorem 2 to bound the solutions of the restricted circular three-body problem near the equilibrium (in case it is Lie stable) over exponentially long times.

First we have to take into account the order $j$ in the normal form (1.7) that determines the Lie stability. When $S=\{\mathbf{0}\}$, then $j=2$ and in the rest of situations $j=4$. Second, we have to consider the number of independent first integrals, that is $d$, which ranges from 1 to 3 in the problem at hand.

In our study the solution is expressed as $\mathbf{I}(t)$, that is a function of the order of $|\mathbf{x}|^{2}$. So, $|\mathbf{x}(0)|<\varepsilon$ implies $|\mathbf{I}(0)|<\varepsilon^{2}$. Introducing $\epsilon=\varepsilon^{2}$ and setting $\mathbf{I}_{0}=\mathbf{I}(0)$, then the thesis of Theorem 2 will read as

$$
|\mathbf{I}(t)|<\alpha \epsilon^{2 / j} \quad \text { for all } t \text { with } \quad 0 \leq t \leq T=\mathcal{C} \exp \left(\frac{\mathcal{E}}{\epsilon^{1 /(2(\nu+1))}}\right),
$$

where $\alpha, \mathcal{C}, \mathcal{E}$ are obtained respectively from $a, C$ and $E$.

Usually for the estimates in terms of action-angle coordinates, the norm used to bound the actions is the 1-norm, but here we use the Euclidean norm as both are equivalent.

The following considerations are in order: 
1. If $S=\{\mathbf{0}\}$, then either $d=2\left(\right.$ cases $\left(a_{2}\right)$ and $\left(b_{2}\right)$ for $\left.\mathbf{k} \notin K\right)$ or $d=3\left(\right.$ case $\left.\left(b_{1}\right)\right)$. Thus, the parameter $\nu$ involved in the Diophantine condition satisfies $\nu \geq 1$ or $\nu \geq 2$, respectively.

When the vector $\sigma=\left(\sigma_{1}, \sigma_{2}\right)$ or $\sigma=\left(\sigma_{1}, \sigma_{2}, \sigma_{3}\right)$ respectively, satisfies (2.2), there exist $\alpha>1$, $\mathcal{C}>0, \mathcal{E}>0$ and $\epsilon_{0}>0$ such that for all $\epsilon \in\left(0, \epsilon_{0}\right)$ with $|\mathbf{I}(0)|<\epsilon$ we get

$$
|\mathbf{I}(t)|<\alpha \epsilon \quad \text { for all } t \text { with } \quad 0 \leq t \leq T=\mathcal{C} \exp \left(\frac{\mathcal{E}}{\epsilon^{1 / 4}}\right),
$$

in $\left(a_{2}\right)$ and $\left(b_{2}\right)$ with $\mathbf{k} \notin K$ or

$$
|\mathbf{I}(t)|<\alpha \epsilon \quad \text { for all } t \text { with } \quad 0 \leq t \leq T=\mathcal{C} \exp \left(\frac{\mathcal{E}}{\epsilon^{1 / 6}}\right),
$$

in the situation $\left(b_{1}\right)$.

2. If $S \neq\{\mathbf{0}\}$, then $j=4$.

- When $d=2, \nu \geq 1$ (cases $\left(b_{2}\right)$ with $\mathbf{k} \in K$ and $\left(b_{3}\right)$ ). Thence, if the frequency vector $\sigma=\left(\sigma_{1}, \sigma_{2}\right)$ satisfies $(2.2)$, there exist $\alpha>1, \mathcal{C}>0, \mathcal{E}>0$ and $\epsilon_{0}>0$ such that for all $\epsilon \in\left(0, \epsilon_{0}\right)$ and $|\mathbf{I}(0)|<\epsilon$, the following estimate holds

$$
|\mathbf{I}(t)|<\alpha \epsilon^{1 / 2} \quad \text { for all } t \text { with } \quad 0 \leq t \leq T=\mathcal{C} \exp \left(\frac{\mathcal{E}}{\epsilon^{1 / 4}}\right) .
$$

- If $d=1$ (case $\left.\left(a_{1}\right)\right)$, then $\nu \geq 0$ and since there is only one first formal integral no Diophantine condition is needed. There exist $\alpha>1, \mathcal{C}>0, \mathcal{E}>0$ and $\epsilon_{0}>0$ such that for all $\epsilon \in\left(0, \epsilon_{0}\right)$ and $|\mathbf{I}(0)|<\epsilon$ we get

$$
|\mathbf{I}(t)|<\alpha \epsilon^{1 / 2} \quad \text { for all } t \text { with } \quad 0 \leq t \leq T=\mathcal{C} \exp \left(\frac{\mathcal{E}}{\epsilon^{1 / 2}}\right) .
$$

Notice that the constants $\alpha, \mathcal{C}$ and $\mathcal{E}$ are independent of $\epsilon$ and are supposed to be obtained using bounds on the normal-form terms.

In summary, we have the following results.

Theorem 4. For the Hamiltonian system associated with (1.1) we have the subsequent asymptotic estimates around the equilibrium point $L_{4}$ in case it is Lie stable:

1. When the vector $\omega$ is associated to a Pythagorean triple in $D$, then there exist $\alpha>1, \mathcal{C}>0$, $\mathcal{E}>0$ and $\epsilon_{0}>0$ such that for all $\epsilon \in\left(0, \epsilon_{0}\right)$ with $|\mathbf{I}(0)|<\epsilon$ :

$$
|\mathbf{I}(t)|<\alpha \epsilon^{1 / 2} \quad \text { for all } t \text { with } \quad 0 \leq t \leq T=\mathcal{C} \exp \left(\frac{\mathcal{E}}{\epsilon^{1 / 2}}\right) .
$$

2. When $\sigma$ is Diophantine, then there exist $\alpha>1, \mathcal{C}>0, \mathcal{E}>0$ and $\epsilon_{0}>0$ such that for all $\epsilon \in\left(0, \epsilon_{0}\right)$ with $|\mathbf{I}(0)|<\epsilon$ :

(a) If either $\omega_{1} \in \mathbb{Q}$ and $\omega_{2} \in \mathbb{R} \backslash \mathbb{Q}$ or $\omega_{1}, \omega_{2} \in \mathbb{R} \backslash \mathbb{Q}$ and there is $\mathbf{k} \notin K$ such that $\mathbf{k} \cdot\left(\omega_{1},-\omega_{2}, 1\right)=0$, then

$$
|\mathbf{I}(t)|<\alpha \epsilon \quad \text { for all } t \text { with } \quad 0 \leq t \leq T=\mathcal{C} \exp \left(\frac{\mathcal{E}}{\epsilon^{1 / 4}}\right) .
$$

(b) If $\omega_{1}, \omega_{2} \in \mathbb{R} \backslash \mathbb{Q}$ and there are no resonances among the main frequencies, then

$$
|\mathbf{I}(t)|<\alpha \epsilon \quad \text { for all } t \text { with } \quad 0 \leq t \leq T=\mathcal{C} \exp \left(\frac{\mathcal{E}}{\epsilon^{1 / 6}}\right) .
$$


(c) If $\omega_{1} \in \mathbb{R} \backslash \mathbb{Q}$ and either $\omega_{2} \in \mathbb{Q}$ or $\omega_{2} \in \mathbb{R} \backslash \mathbb{Q}$ and there is $\mathbf{k} \in K$ such that $\mathbf{k}$. $\left(\omega_{1},-\omega_{2}, 1\right)=0$, then

$$
|\mathbf{I}(t)|<\alpha \epsilon^{1 / 2} \quad \text { for all } t \text { with } \quad 0 \leq t \leq T=\mathcal{C} \exp \left(\frac{\mathcal{E}}{\epsilon^{1 / 4}}\right) .
$$

We specify the results obtained by our approach for some values handled in previous studies.

Corollary 1. Given $\epsilon \in\left(0, \epsilon_{0}\right)$ and $|\mathbf{I}(0)|<\epsilon$, the following estimates around the equilibrium point $L_{4}$ are satisfied.

For $\mu=\mu_{1}, \mu_{2}$ we obtain

$$
|\mathbf{I}(t)|<\alpha \epsilon \quad \text { for all } t \text { with } \quad 0 \leq t \leq T=\mathcal{C} \exp \left(\frac{\mathcal{E}}{\epsilon^{1 / 6}}\right) .
$$

For $\mu=\mu_{(3,3,-2)}$ we achieve

$$
|\mathbf{I}(t)|<\alpha \epsilon \quad \text { for all } t \text { with } \quad 0 \leq t \leq T=\mathcal{C} \exp \left(\frac{\mathcal{E}}{\epsilon^{1 / 4}}\right) .
$$

For $\mu=\mu_{(0,3,1)}$ we arrive at

$$
|\mathbf{I}(t)|<\alpha \epsilon^{1 / 2} \quad \text { for all } t \text { with } \quad 0 \leq t \leq T=\mathcal{C} \exp \left(\frac{\mathcal{E}}{\epsilon^{1 / 4}}\right) .
$$

For $\mu=\mu_{3} \approx 0.014780913055964$ we get

$$
|\mathbf{I}(t)|<\alpha \epsilon \quad \text { for all } t \text { with } \quad 0 \leq t \leq T=\mathcal{C} \exp \left(\frac{\mathcal{E}}{\epsilon^{1 / 6}}\right) .
$$

The case $\mu_{3}$ will be tackled in section 5 .

\section{COMPARISON WITH PREVIOUS RESULTS}

Regarding related approaches to the nonlinear stability of $L_{4}$, we mention that in 1971 Markeev [20] proved the stability of $L_{4}$ in $\mu \in\left(0, \mu_{R}\right) \backslash\left\{\mu_{(1,2,0)}, \mu_{(1,3,0)}\right\}$ for most initial conditions, in the sense of the Lebesgue measure. In other words, he proved the existence of KAM 3tori around $L_{4}$. In 1973, see [21], the same author proved the formal stability of $L_{4}$ for $\mu \in$ $\left(0, \mu_{1}\right) \cup\left(\mu_{2}, \mu_{(1,2,0)}\right) \cup\left(\mu_{(1,2,0)}, \mu_{R}\right)$. This corresponds to the domain $D$ that we have defined in (3.3). The formal stability analysis made in $\left(\mu_{1}, \mu_{2}\right)$ is not complete as double resonances are not considered for orders higher than 6 .

Remark 3. Theorem 3 recovers the formal stability achievements already obtained in the set $D$ and extends them to $\mu_{1}, \mu_{2}$ and the values $\mu \in\left(\mu_{1}, \mu_{2}\right)$ not leading to a Pythagorean triple. Moreover our proof of Lie stability uses different arguments from the one of Markeev in [21]. Indeed, our approach is straightforward since we only need that $\mathcal{H}^{4}$ restricted to the set $S$ be sign-definite, while Markeev introduced a formal first integral in each specific case.

In 1989, Giorgilli et al. [14], applying normal form techniques with floating-point arithmetic, proved that $L_{4}$ was Nekhoroshev stable for $\mu \in\left(0, \mu_{R}\right)$, excepting a few values of $\mu$ that led to resonances. Furthermore the vector $\omega$ should satisfy a Diophantine condition. In 1991, Celletti and Giorgilli [8], along the same line, refined the preceding achievements.

In 1998 Benettin et al. [3] extended previous results with the idea of determining Nekhoroshev stability without imposing any Diophantine condition. On the one hand, the requirement of quasiconvexity was relaxed by introducing the concept of directional quasi-convexity, that is specific for elliptic equilibria. On the other hand, they enlarged the directional quasi-convexity requirement by proposing a steepness condition on the 3 -jet of the sixth order normal form, following the original ideas of Nekhoroshev. They applied their results using normal forms with floating-point arithmetic to establish that $\mathcal{H}^{4}(\mathbf{I})$ was directionally quasi-convex, and then concluding that $L_{4}$ is Nekhoroshev stable in the domain $D$. 
Remark 4. It is worth mentioning that the estimates claimed in Theorem 2 of [3] have not been proved so far, and only very recently bounds for steep elliptic equilibria have appeared [4]. As these estimates depend on the steepness indices, it is not immediate to apply them in our problem, therefore we have preferred to use the ones of [3] in our comparisons.

For $\mu \in D,|\mathbf{I}(0)| \leq \epsilon$ and $\epsilon$ sufficiently small, two estimates were obtained in [3], namely,

$$
|\mathbf{I}(t)| \leq \epsilon^{1 / 3} \quad \text { for } \quad 0<\mathrm{t} \leq \exp \left(\epsilon^{-1 / 3}\right)
$$

and

$$
|\mathbf{I}(t)| \leq \epsilon^{1 / 2} \quad \text { for } \quad 0<\mathrm{t} \leq \exp \left(\epsilon^{-1 / 6}\right) .
$$

Several comparisons between our approach and that of [3], when $\omega_{1}$ is in the domain $D$, are in order:

(i) According to Theorem 4, when $\omega$ is associated to a Pythagorean triple in $D$, our confinement bound for $|\mathbf{I}(t)|$ is of the same order $\epsilon^{1 / 2}$ but our time estimate is longer, indeed of the order of $\exp \left(\epsilon^{-1 / 2}\right)$, whereas in (5.2) is $\exp \left(\epsilon^{-1 / 6}\right)$.

(ii) When the vector $\sigma$ satisfies the Diophantine condition (2.2) and $S=\{\mathbf{0}\}$ the bounds obtained using Theorem 4 are sharper than the ones of (5.2). Observe that in cases 2.(a) and 2.(b) of Theorem 4 the confinement is of order $\epsilon$, whereas in (5.1) and (5.2) it is either of order $\epsilon^{1 / 2}$ or $\epsilon^{1 / 3}$. In fact, the order $\epsilon$ is stated in the context of Nekhoroshev estimates only when the 3 -jet is computed.

(iii) Under the usual Diophantine condition, in case 2.(c) of Theorem 4, the time estimate is better than it is in (5.2), say $\exp \left(\epsilon^{-1 / 4}\right)$ versus $\exp \left(\epsilon^{-1 / 6}\right)$, for the same confinement of the solution, that is $\epsilon^{1 / 2}$, using only the normal form $\mathcal{H}^{4}$, that is, without calculating higher-order terms.

When $\mu \in\left[\mu_{1}, \mu_{2}\right]$, Benettin et al. [3] calculated the normal form term $\mathcal{H}^{6}(\mathbf{I})$ (with numerical coefficients) as directional quasi-convexity does not hold in this interval. It was also checked that the normal form of order eight could be computed excepting the values $\mu_{(1,3,0)}, \mu_{(0,3,1)}$ and $\mu_{(3,3,-2)}$. They also proved that $\mathcal{H}^{6}(\mathbf{I})$ was steep except at $\mu_{3}$. More specifically, they determined when $\mathcal{H}^{6}$ was non-degenerate, in other words, under which conditions the unique solution of $\mathcal{H}_{2}(\mathbf{I})=\mathcal{H}_{4}(\mathbf{I})=\mathcal{H}_{6}(\mathbf{I})=0$ was $\mathbf{I}=\mathbf{0}$. To simplify the calculations, it was also required in [3] that the restriction of the Hessian matrix of $\mathcal{H}_{4}$, say $A$, to the plane orthogonal to $\omega$ is nonsingular, in other words if $\omega \cdot \mathbf{I}=0, A \mathbf{I}=\mathbf{0}$ then $\mathbf{I}=\mathbf{0}$. Then, excluding the aforementioned values, steepness (and Nekhoroshev stability) was concluded in $\left[\mu_{1}, \mu_{2}\right]$ and the estimates were as follows.

For $\mu \in\left[\mu_{1}, \mu_{2}\right] \backslash\left\{\mu_{(3,3,-2)}, \mu_{(1,3,0)}, \mu_{3}, \mu_{(0,3,1)}\right\},|\mathbf{I}(0)| \leq \epsilon$ and $\epsilon$ small enough:

$$
|\mathbf{I}(t)|<\epsilon \text { for } 0<\mathrm{t}<\exp \left(\epsilon^{-1 / 20}\right) .
$$

The cases $\mu_{(3,3,-2)}, \mu_{(0,3,1)}$ were excluded from the estimates (5.3) because, although for both cases $\mathcal{H}_{6}$ depends only on the actions, the corresponding $\mathcal{H}_{8}$ contains resonant terms, and the above bounds require that $\mathcal{H}^{8}$ depend only on $\mathbf{I}$.

In the interval $\left[\mu_{1}, \mu_{2}\right]$ we stress the following points of our approach:

(i) For $\mu \in\left[\mu_{1}, \mu_{2}\right] \backslash\left\{\mu_{(3,3,-2)}, \mu_{(1,3,0)}, \mu_{3}, \mu_{(0,3,1)}\right\}$, when $\sigma$ satisfies (2.2), and such that the frequencies leading to Pythagorean triples are excluded, the time estimates of Theorem 4 obtained through $\mathcal{H}^{4}$ are better than the ones in (5.3) deduced from $\mathcal{H}^{6}$. Nevertheless, the confinement of the actions obtained in [3] is sharper, excepting when $S=\{\mathbf{0}\}$, as then both are of the same order. 
(ii) The case of the Pythagorean triples for $\mu \in\left(\mu_{1}, \mu_{2}\right)$ is a pending issue in our analysis. What happens is that $\mathcal{H}_{4}$ changes sign in $S$ and then, we cannot conclude Lie stability. According to (B) in Theorem 1, computing higher-order terms in the normal form would not lead to a sign-definite formal integral. From [3] (and from our own analysis) we know that $\mathcal{H}_{6}$ is steep for all Pythagorean triples, thus bounds for the solution are provided. Although steepness does not imply stability, if instability holds, the diffusion mechanism would be very slow.

(iii) Case $\mu_{(3,3,-2)}$ is Lie stable, as it belongs to $\left(b_{2}\right)$ in section 3. Case $\mu_{(0,3,1)}$ is also Lie stable, as it belongs to $\left(b_{3}\right)$ in section 3 . Corollary 1 gives asymptotic estimates in both cases. However they cannot be studied from the point of view of Nekhoroshev theory.

(iv) Cases $\mu_{(1,3,0)}$ and $\mu_{(1,2,0)}$ do not satisfy the necessary conditions leading to Nekhoroshev stability, but they are already known to be unstable.

The case $\mu_{3}$ corresponds to a degenerate 3 -jet. So, when applying Nekhoroshev techniques, one needs to pursue the calculations to get the 4 -jet, that is, $\mathcal{H}^{8}(\mathbf{I})$. Moreover, one has to take into account that for $r$-jets with $r>3$ additional conditions apart from the non-degeneracy of the jet are needed to ensure steepness. This case was studied by Schirinzi and Guzzo in 2015 [27], establishing Nekhoroshev stability for $\mu=\mu_{3}$. It should be noticed that their normal form calculations were performed using floating-point arithmetic. However, the related asymptotic estimates have not been determined so far.

Trying to get the value $\mu_{3}$ from our normal form $\mathcal{H}^{6}$ we notice that the corresponding 3-jet becomes zero for $\mu_{3}$ but then the associated $I_{3}<0$. More precisely, in our analysis $\mu_{3}$ does not appear as a special value and Lie stability is accomplished without the need of analysing $\mathcal{H}^{6}$. The value $\mu_{3} \approx 0.014780913055963$ is in correspondence through (1.3) with the value $\omega_{1}$ lying in the interval $\left(\omega_{1}^{\sharp}, \omega_{1}^{*}\right)$ obtained as the square root of a root of the polynomial of degree 52 given by $\sum_{i=0}^{52} c_{i} x^{i}$ whose coefficients are given in Table 3 of the Appendix. By applying the rational root test we have proved that the value of $\omega_{1}$ related to $\mu_{3}$ is irrational and the same is true with the corresponding $\omega_{2}$. Therefore this situation is a specific example of $\left(b_{1}\right)$ in section 3.

We do the following considerations regarding $\mu_{3}$ :

(i) For $\mu_{3}$ the equilibrium is Lie stable and time estimates are given in Corollary 1. This conclusion is obtained just from the analysis of $\mathcal{H}^{4}$.

(ii) The calculations we have performed in order to achieve Lie stability use symbolic arithmetic. We have carried out the computations up to order 4 , that is, determining $\mathcal{H}^{6}(\mathbf{I})$, although for our analysis only order 2 is required, excepting for the explicit calculation of $\mu_{3}$. The computations are valid disregarding the resonances that appear in the generating functions and that we have treated separately.

It is not straightforward to compare our asymptotic estimates with those obtained in [3] and other references, but in general our bounds are sharper than those accomplished with the use of Nekhoroshev theory with low order normal forms. For instance, using $\mathcal{H}^{4}$ in the Lie stable cases where $\operatorname{dim} S=2$ our time estimate is of the order $\exp \left(\mathcal{E} \epsilon^{-1 / 2}\right)$, which is not obtained applying Nekhoroshev theory. Moreover, the exponent of $\epsilon^{-1}$ in the time estimates never exceeds $1 / 6$, while in [3] it can be $1 / 20$, as we have seen before. Sharper bounds could be obtained in the setting of Nekhoroshev theory by computing higher-order normal forms, see for instance [3, 26], and references therein.

Finally we point out a couple of considerations related to our estimates: 
(i) It would be desirable to drop, or at least to relax, the Diophantine condition in Theorem 2, but so far it is not possible since it is an essential requirement for estimating the bounds for formal integrals in [9]. Sometimes given a non-resonant vector $\sigma$ it is not straightforward to deduce whether it is Diophantine or Liouville, although it is a well-known fact that for a fixed $\nu$ the Lebesgue measure of the set of vectors $\sigma \in \mathbb{R}^{d}$ that does not satisfy the Diophantine condition for any $c>0$ is zero. In cases of Liouville vectors $\sigma$ we make use of Benettin et al.'s bounds given in (5.1) and (5.2). In this context, perhaps we could apply Lochak's method of averaging [18] by analysing the neighbourhoods of periodic solutions of the unperturbed system, see reference [26], dropping therefore the need of using condition (2.2).

(ii) In case $\left(b_{1}\right)$ it could happen that the frequency vector $\sigma=\left(\sigma_{1}, \sigma_{2}, \sigma_{3}\right)$ be Diophantine. If this occurs the best time estimates are of doubly exponential character, see [5].

In Fig. 1 we adapt Fig. 1 from [3] to collect known results about the stability of $L_{4}$.

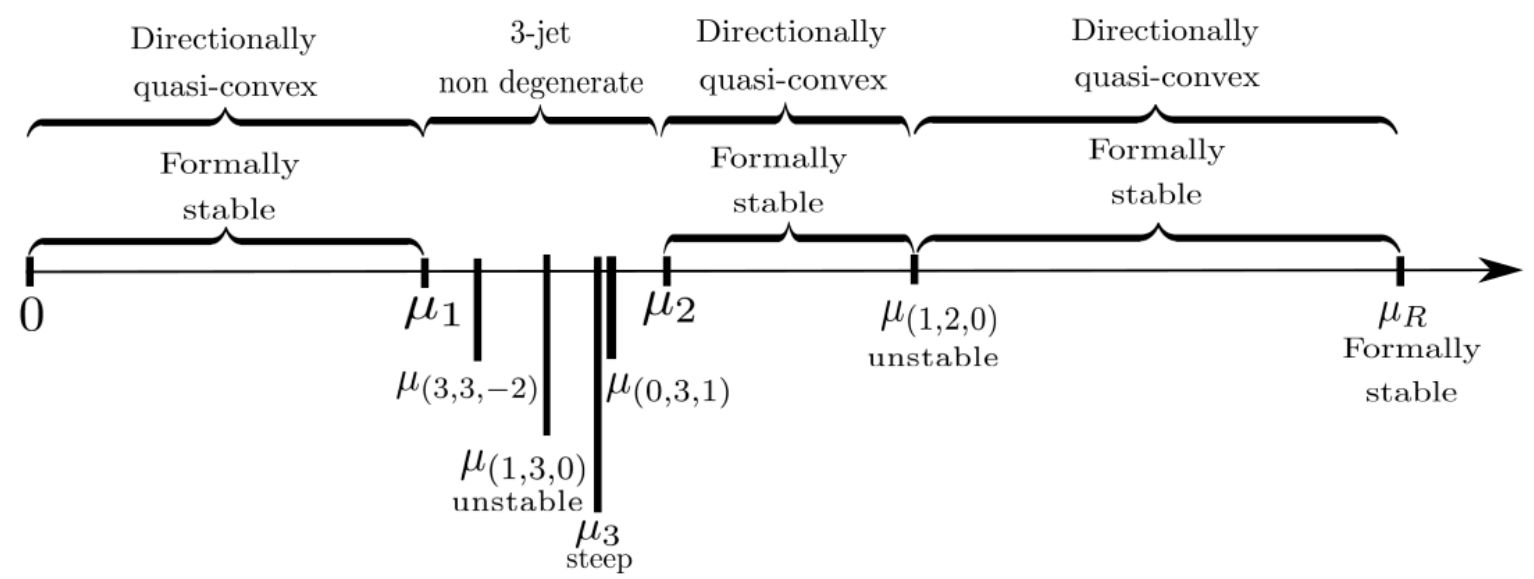

Fig. 1. Type of stability of the Lagrangian points depending on $\mu$ in the spatial case.

\section{KAM TORI}

In this section we prove the existence of 3-dimensional KAM tori and quasi-periodic motions encasing the equilibrium point $L_{4}$ of the Hamiltonian system related to (1.1) at each energy level. This is indeed Markeev's analysis performed in [20]. For our purpose we apply the classical theorem by Kolmogorov, Arnold and Moser in its isoenergetic version, see, for instance [1]. Thus, an isoenergetic nondegeneracy condition has to be satisfied. More precisely, we get

$$
D_{4}=\left|\begin{array}{cc}
\frac{\partial^{2} \mathcal{H}^{4}}{\partial \mathbf{I}^{2}} & \frac{\partial \mathcal{H}^{4}}{\partial \mathbf{I}} \\
\frac{\partial \mathcal{H}^{4}}{\partial \mathbf{I}} & 0
\end{array}\right|=\frac{\omega_{1}^{2}\left(\omega_{1}^{2}-1\right) d_{4}}{559872\left(1-2 \omega_{1}^{2}\right)^{6}\left(\omega_{1}^{2}-4\right)^{3}\left(\omega_{1}^{2}+3\right)^{3}\left(5 \omega_{1}^{2}-4\right)^{3}\left(5 \omega_{1}^{2}-1\right)^{3}},
$$

where

$$
\begin{aligned}
d_{4}= & -856968120000 \omega_{1}^{32}+6855744960000 \omega_{1}^{30}-12012443413200 \omega_{1}^{28}-35888432907600 \omega_{1}^{26} \\
& +156438442275660 \omega_{1}^{24}-160144630175160 \omega_{1}^{22}+31637715760125 \omega_{1}^{20} \\
& -362513394226125 \omega_{1}^{18}+1355837182686882 \omega_{1}^{16}-2073003173172738 \omega_{1}^{14} \\
& +1782670403156769 \omega_{1}^{12}-952761177324729 \omega_{1}^{10}+327497353333812 \omega_{1}^{8} \\
& -74195783114400 \omega_{1}^{6}+11875034325888 \omega_{1}^{4}-1435874045184 \omega_{1}^{2}+95785141248 .
\end{aligned}
$$




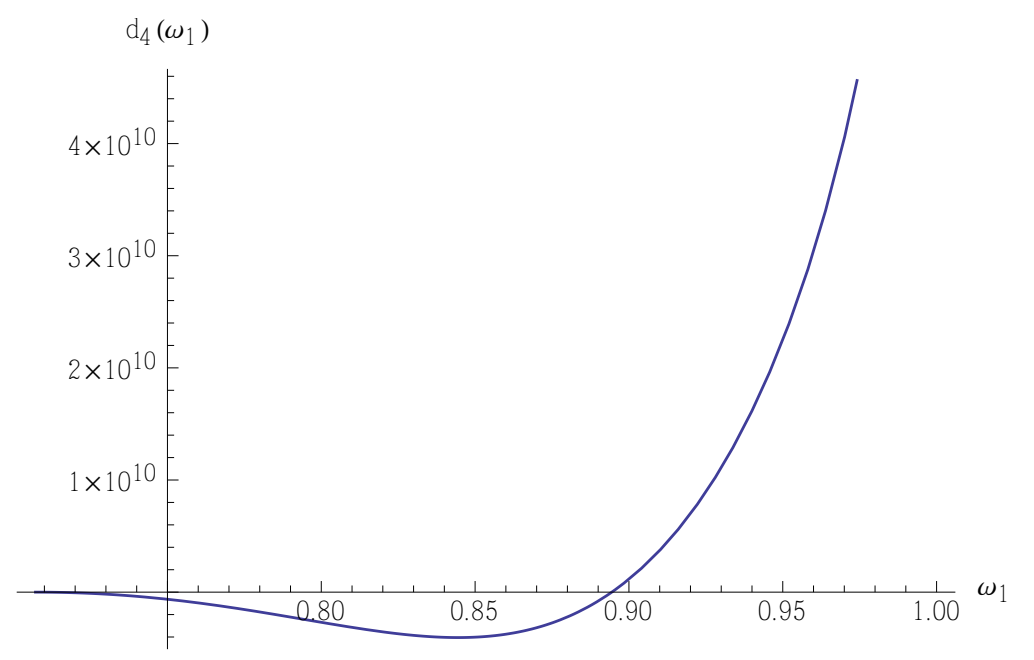

Fig. 2. Graph of the curve $d_{4}\left(\omega_{1}\right)$.

Note that the function $d_{4}=d_{4}\left(\omega_{1}\right)$ is null only when $\omega_{1}=2 / \sqrt{5}$, i.e., $\mu=\mu_{(1,2,0)}$, see Fig. 2 .

Thus, $D_{4}$ does not vanish for $\omega_{1} \in(1 / \sqrt{2}, 1) \backslash\{2 / \sqrt{5}\}$. Even in the situations where we do not know if Lie stability holds, that is, the ones corresponding with Pythagorean triples such that $\mu \in\left(\mu_{1}, \mu_{2}\right), D_{4}$ is different from zero.

We establish the following result.

Theorem 5. For $\mu \in\left(0, \mu_{R}\right) \backslash\left\{\mu_{(1,2,0)}, \mu_{(1,3,0)}\right\}$, most of the invariant 3-tori corresponding to the term $\mathcal{H}^{4}$ derived from the Hamiltonian system related to (1.1) will persist slightly deformed for any sufficiently small perturbation of them, that is, for the full system. Moreover, the Lebesgue measure of the complement of the set of tori tends to zero when the perturbation is small. More precisely, the invariant tori form a majority on each energy-level manifold. The measure of the complement of the invariant tori that remain is of the order $\mathcal{O}\left(\varepsilon^{1 / 4}\right)$ and can be refined to $\mathcal{O}\left(\varepsilon^{(l-3) / 4}\right)$ when the frequency vector $\omega$ does not satisfy resonance relations of order $l$ with $l \leq 4$.

Remark 5. We have also applied Han-Li-Yi's Theorem [15] dropping the isoenergetic condition. In this way we have obtained invariant 3 -tori and quasiperiodic motions for the full Hamiltonian of the spatial circular restricted three body problem (1.1) in the situations where it can be expressed as $H_{2}(\mathbf{I})+\varepsilon^{2} \mathcal{H}_{4}(\mathbf{I})+\mathcal{O}\left(\varepsilon^{4}\right)$. Around $L_{4}$, this is possible after introducing a stretching of coordinates as we have done in the course of the paper, discarding the two unstable cases, for a sufficiently small $\varepsilon>0$. Specifically there are families of invariant 3-tori enclosing the equilibrium point $L_{4}$. These invariant tori form a majority in the sense that the measure of the complement of their union is of the order $\mathcal{O}\left(\varepsilon^{\delta}\right)$ with a fixed value of $\delta$ such that $0<\delta<1 / 5$. The invariant tori are organised in Cantor families that depend on the parameter $\mu$.

Notice that the application of Han-Li-Yi's Theorem for high-order proper degeneracy yields similar results to the ones obtained through Thereom 5. Indeed, according to Han et al., the measure of the tori that do not remain after the perturbation can be refined pushing the normal form computation to higher orders in case that resonant terms are not encountered, analogously as in Theorem 5 .

\section{APPENDIX}

We include two tables containing the single resonant cases up to $|\mathbf{k}|_{1}=8$. Table 1 accounts for the case of $\operatorname{dim} S=0$. For the case of $\operatorname{dim} S=1$, see Table 2 .

In Table 3 we give the coefficients of the polynomial $\mu_{3}$ comes from as a root. 


\begin{tabular}{|l|l|l|l|l|}
\hline $\mathbf{k}$ & $F_{1}$ & $F_{2}$ & $\sigma=\left(\sigma_{1}, \sigma_{2}\right)$ & Case \\
\hline$(2,2,-1)$ & $I_{2}-I_{1}$ & $I_{1}+2 I_{3}$ & $\left(\frac{1-\sqrt{7}}{4}, \frac{1}{2}\right)$ & $\left(b_{2}\right)$ \\
\hline$(3,2,-1)$ & $3 I_{2}-2 I_{1}$ & $I_{1}+3 I_{3}$ & $\left(\frac{2(1-3 \sqrt{3})}{39}, \frac{1}{3}\right)$ & $\left(b_{2}\right)$ \\
\hline$(3,1,-2)$ & $3 I_{2}-I_{1}$ & $2 I_{1}+3 I_{3}$ & $\left(\frac{2-3 \sqrt{6}}{30}, \frac{1}{3}\right)$ & $\left(b_{2}\right)$ \\
\hline$(2,3,-1)$ & $2 I_{2}-3 I_{1}$ & $I_{1}+2 I_{3}$ & $\left(\frac{3-4 \sqrt{3}}{26}, \frac{1}{2}\right)$ & $\left(b_{2}\right)$ \\
\hline$(1,-3,-2)$ & $3 I_{1}+I_{2}$ & $2 I_{1}+I_{3}$ & $\left(\frac{-6+\sqrt{6}}{10}, 1\right)$ & $\left(b_{2}\right)$ \\
\hline$(4,0,-3)$ & $I_{2}$ & $3 I_{1}+4 I_{3}$ & $\left(-\frac{\sqrt{7}}{4}, \frac{1}{4}\right)$ & $\left(a_{2}\right)$ \\
\hline$(3,3,-1)$ & $I_{2}-I_{1}$ & $I_{1}+3 I_{3}$ & $\left(\frac{1-\sqrt{17}}{6}, \frac{1}{3}\right)$ & $\left(b_{2}\right)$ \\
\hline$(2,4,-1)$ & $I_{2}-2 I_{1}$ & $I_{1}+2 I_{3}$ & $\left(\frac{2-\sqrt{19}}{10}, \frac{1}{2}\right)$ & $\left(b_{2}\right)$ \\
\hline$(1,-4,-2)$ & $4 I_{1}+I_{2}$ & $2 I_{1}+I_{3}$ & $\left(\frac{-8+\sqrt{13}}{17}, 1\right)$ & $\left(b_{2}\right)$ \\
\hline$(4,3,-1)$ & $4 I_{2}-3 I_{1}$ & $I_{1}+4 I_{3}$ & $\left(\frac{3-8 \sqrt{6}}{100}, \frac{1}{4}\right)$ & $\left(b_{2}\right)$ \\
\hline$(4,1,-3)$ & $4 I_{2}-I_{1}$ & $3 I_{1}+4 I_{3}$ & $\left(\frac{3-8 \sqrt{2}}{68}, \frac{1}{4}\right)$ & $\left(b_{2}\right)$ \\
\hline$(3,4,-1)$ & $3 I_{2}-4 I_{1}$ & $I_{1}+3 I_{3}$ & $\left(\frac{2(2-3 \sqrt{6})}{75}, \frac{1}{3}\right)$ & $\left(b_{2}\right)$ \\
\hline$(3,3,-2)$ & $I_{1}-I_{2}$ & $2 I_{1}+3 I_{3}$ & $\left(\frac{-2+\sqrt{14}}{6}, \frac{1}{3}\right)$ & $\left(b_{2}\right)$ \\
\hline$(2,5,-1)$ & $2 I_{2}-5 I_{1}$ & $I_{1}+2 I_{3}$ & $\left(\frac{5-4 \sqrt{7}}{58}, \frac{1}{2}\right)$ & $\left(b_{2}\right)$ \\
\hline$(1,-4,-3)$ & $4 I_{1}+I_{2}$ & $3 I_{1}+I_{3}$ & $\left(\frac{2(-6+\sqrt{2})}{17}, 1\right)$ & $\left(b_{2}\right)$ \\
\hline$(1,-5,-2)$ & $5 I_{1}+I_{2}$ & $2 I_{1}+I_{3}$ & $\left(\frac{-10+\sqrt{22}}{26}, 1\right)$ & $\left(b_{2}\right)$ \\
\hline$(3,0)$ & & & \\
\hline
\end{tabular}

Table 1. Resonance vector, first integrals and vector $\sigma$ in cases of $\operatorname{dim} S=0$. Hamiltonian $H_{2}=$ $\sigma_{1} F_{1}+\sigma_{2} F_{2}$. In the situation $\left(b_{2}\right), \mathbf{k} \notin K$.

\section{ACKNOWLEDGMENTS}

The present paper is part of the thesis of the first author [6]. The comments provided by the referees have helped us improve a first version of the paper.

\section{FUNDING}

The authors are partially supported by Project MTM 2017-88137-C2-1-P of the Ministry of Science, Innovation and Universities of Spain. D. Cárcamo-Díaz acknowledges support from CONICYT PhD/2016-21161143. C. Vidal is partially supported by Fondecyt grant 1180288 .

\section{CONFLICT OF INTEREST}

The authors declare that they have no conflicts of interest. 


\begin{tabular}{|c|c|c|c|c|}
\hline k & $F_{1}$ & $F_{2}$ & $\sigma=\left(\sigma_{1}, \sigma_{2}\right)$ & Case \\
\hline$(0,2,1)$ & $I_{1}$ & $I_{2}-2 I_{3}$ & $\left(\frac{\sqrt{3}}{2},-\frac{1}{2}\right)$ & $\left(b_{3}\right)$ \\
\hline$(0,3,1)$ & $I_{1}$ & $I_{2}-3 I_{3}$ & $\left(\frac{2 \sqrt{2}}{3},-\frac{1}{3}\right)$ & $\left(b_{3}\right)$ \\
\hline$(2,3,0)$ & $3 I_{1}-2 I_{2}$ & $I_{3}$ & $\left(\frac{1}{\sqrt{13}}, 1\right)$ & $\left(b_{2}\right)$ \\
\hline$(0,4,1)$ & $I_{1}$ & $I_{2}-4 I_{3}$ & $\left(\frac{\sqrt{15}}{4},-\frac{1}{4}\right.$ & $\left(b_{3}\right)$ \\
\hline$(0,3,2)$ & $I_{1}$ & $2 I_{2}-3 I_{3}$ & $\left(\frac{\sqrt{5}}{3},-\frac{1}{3}\right)$ & $\left(b_{3}\right)$ \\
\hline$(1,5,0)$ & $5 I_{1}-I_{2}$ & $I_{3}$ & $\left(\frac{1}{\sqrt{26}}, 1\right)$ & $\left(b_{2}\right)$ \\
\hline$(0,5,1)$ & $I_{1}$ & $I_{2}-5 I_{3}$ & $\left(\frac{2 \sqrt{6}}{5},-\frac{1}{5}\right.$ & $\left(b_{3}\right)$ \\
\hline$(2,5,0)$ & $5 I_{1}-2 I_{2}$ & $I_{3}$ & $\left(\frac{1}{\sqrt{29}}, 1\right)$ & $\left(b_{2}\right)$ \\
\hline$(2,4,1)$ & $2 I_{1}-I_{2}$ & $I_{1}-2 I_{3}$ & $\left(\frac{2+\sqrt{19}}{10},-\frac{1}{2}\right)$ & $\left(b_{2}\right)$ \\
\hline$(1,6,0)$ & $6 I_{1}-I_{2}$ & $I_{3}$ & $\left(\frac{1}{\sqrt{37}}, 1\right)$ & $\left(b_{2}\right)$ \\
\hline$(1,4,2)$ & $4 I_{1}-I_{2}$ & $2 I_{1}-I_{3}$ & $\left(\frac{\sqrt{13}+8 \sqrt{17}}{17},-\sqrt{17}\right)$ & $\left(b_{2}\right)$ \\
\hline$(0,6,1)$ & $I_{1}$ & $I_{2}-6 I_{3}$ & $\left(\frac{\sqrt{35}}{6},-\frac{1}{6}\right)$ & $\left(b_{3}\right)$ \\
\hline$(0,5,2)$ & $I_{1}$ & $2 I_{2}-5 I_{3}$ & $\left(\frac{\sqrt{21}}{5},-\frac{1}{5}\right)$ & $\left(b_{3}\right)$ \\
\hline$(3,5,0)$ & $5 I_{1}-3 I_{2}$ & $I_{3}$ & $\left(\frac{1}{\sqrt{34}}, 1\right)$ & $\left(b_{2}\right)$ \\
\hline$(2,5,1)$ & $5 I_{1}-2 I_{2}$ & $I_{1}-2 I_{3}$ & $\left(\frac{5+4 \sqrt{7}}{58},-\frac{1}{2}\right)$ & $\left(b_{2}\right)$ \\
\hline$(1,7,0)$ & $7 I_{1}-I_{2}$ & $I_{3}$ & $\left(\frac{1}{5 \sqrt{2}}, 1\right)$ & $\left(b_{2}\right)$ \\
\hline$(1,5,2)$ & $5 I_{1}-I_{2}$ & $2 I_{1}-I_{3}$ & $\left(\frac{10+\sqrt{22}}{26},-1\right)$ & $\left(b_{2}\right)$ \\
\hline$(0,7,1)$ & $I_{1}$ & $I_{2}-7 I_{3}$ & $\left(\frac{4 \sqrt{3}}{7},-\frac{1}{7}\right)$ & $\left(b_{3}\right)$ \\
\hline
\end{tabular}

Table 2. Resonance vector, first integrals and vector $\sigma$ in cases of $\operatorname{dim} S=1$. Hamiltonian $H_{2}=$ $\sigma_{1} F_{1}+\sigma_{2} F_{2}$. In the situation corresponding to $\left(b_{2}\right), \mathbf{k} \in K$.

\section{REFERENCES}

1. Arnol'd, V.I., Kozlov, V.V. and Neishtadt, A.I., Mathematical aspects of classical and celestial mechanics, Dynamical Systems III Encyclopaedia of Mathematical Sciences 3, 3rd ed., Berlin: SpringerVerlag, 2006.

2. Bardin, B., On Motions Near the Lagrange Equilibrium Point $L_{4}$ in the Case of Routh's Critical Mass Ratio, Celestial Mech. Dynam. Astronom., 2002, vol. 82, no. 2, pp.163-177. 


\begin{tabular}{|c|c|c|c|}
\hline$c_{0}$ & -33455436474446988725240946644484096 & $c_{1}$ & 3194933800193210510939874224007806976 \\
\hline$c_{2}$ & -133142852751634975765240694606860910592 & $c_{3}$ & 3353229252229449617635906930441300475904 \\
\hline$c_{4}$ & -59006295355549187747044109281616236118016 & $c_{5}$ & 788225115948882812404191577579711057035264 \\
\hline$c_{6}$ & -8404704358510620010977067016337185034731520 & $c_{7}$ & 73695475954794906843167530358753671462404096 \\
\hline$c_{8}$ & -540576732470886723756654268885633469206699008 & $c_{9}$ & 3350560634073938838006800472295740136106555136 \\
\hline$c_{10}$ & -17657500682256413919143297554372385724778097280 & $c_{11}$ & 79463164160925835810094642601972732662428571232 \\
\hline$c_{12}$ & -306396477115452068307283022024953343053130416596 & $c_{13}$ & 1015297587226646281157210009476933721162552276229 \\
\hline$c_{14}$ & -2901167315298202702512087053641668436785252459945 & $c_{15}$ & 7184430538308699052301532759394960483420936688598 \\
\hline$c_{16}$ & -15552532804758882888717376187454633135733711386930 & $c_{17}$ & 29880956548317259685613622218904331089896620336199 \\
\hline$c_{18}$ & -52201223121743383064233227580596228239865365448051 & $c_{19}$ & 85510386721294691989215784315942923230159418394628 \\
\hline$c_{20}$ & -134625043875319416149393738742778612287835778168608 & $c_{21}$ & 203983737378961879679113646763821294842098948776483 \\
\hline$c_{22}$ & -290058963748297439559277326592130302207012032103487 & $c_{23}$ & 372821687408889124493528843631412931581817725164518 \\
\hline$c_{24}$ & -417681073832456682793620148713018873912445009123434 & $c_{25}$ & 394397633015250251046398071718980958400736933611921 \\
\hline$c_{26}$ & -301566539207032698480846971131018825843193755572069 & $c_{27}$ & 173540952413898297286246299380019891424924554663552 \\
\hline$c_{28}$ & 04615639896711720030183726 & $c_{29}$ & -7679677151122149928520105697347585943096732332960 \\
\hline$c_{30}$ & 2761398966659 & $c_{31}$ & -20980601034714219218681474857905315558796080240640 \\
\hline$c_{32}$ & 920 & $c_{33}$ & -2345627974195951773973005090446131999866171647232 \\
\hline$c_{34}$ & 468644966788218481020647383542439007628150846720 & $c_{35}$ & -530135429423849146464518754398950077120430964736 \\
\hline$c_{36}$ & 639827077256385977476978518239705975042078466048 & $c_{37}$ & -472355628562578539510969144175875192178435473408 \\
\hline$c_{38}$ & 246133207108175370094381015583635396587927519232 & $c_{39}$ & -99872439676762163536197196055142095448444108800 \\
\hline$c_{40}$ & 33952315204186600296511625347287194679262576640 & $c_{41}$ & -10271715872598767663299743179381342718579507200 \\
\hline$c_{42}$ & 2867337640268954204120843075629828505228083200 & $c_{43}$ & -737859950885920377593058895165940280852480000 \\
\hline$c_{44}$ & 170062953704533146451372328358210842787840000 & $c_{45}$ & -34201507579437555095782374809247055872000000 \\
\hline$c_{46}$ & 6116590633655045522465092353724350464000000 & $c_{47}$ & -1064226850447741909301401361239244800000000 \\
\hline$c_{48}$ & 191742496293562199236270016803635200000000 & $c_{49}$ & -31995403391989269030444412928000000000000 \\
\hline$c_{50}$ & 4069107976732780819991272325120000000000 & $c_{51}$ & -328151981300377653973352448000000000000 \\
\hline$c_{52}$ & 12621230050014525152821248000000000000 & & \\
\hline
\end{tabular}

Table 3. Coefficients of the polynomial $\sum_{i=0}^{52} c_{i} x^{i}$ from where $\mu_{3}$ comes as a root.

3. Benettin, G., Fassò, F. and Guzzo, M., Nekhoroshev-Stability of $L_{4}$ and $L_{5}$ in the Spatial Restricted Three-Body Problem, Regul. Chaotic Dyn., 1998, vol. 3, no. 3, pp. 56-71.

4. Bounemoura, A., Fayad, B. and Niederman, L., Nekhoroshev Estimates for Steep Real-Analytic Elliptic Equilibrium Points, Nonlinearity, 2020, vol. 33, no. 1, pp.1-33.

5. Bounemoura, A., Fayad, B. and Niederman, L., Super-Exponential Stability for Generic Real-Analytic Elliptic Equilibrium Points, hal-01188980v2, 2019.

6. Cárcamo-Díaz, D., Stability and Estimates Near Elliptic Equilibrium Points in Hamiltonian Systems and Applications, PhD Thesis, Universidad del Bío-Bío, Concepción, Chile, 2019.

7. Cárcamo-Díaz, D., Palacián, J. F., Vidal, C. and Yanguas, P., Formal Stability of Elliptic Equilibria in Hamiltonian Systems with Exponential Time Estimates, Preprint, 2019.

8. Celletti, A. and Giorgilli, A., On the Stability of the Lagrangian Points in the Spatial Restricted Problem of Three Bodies, Celestial Mech. Dynam. Astronom., 1991, vol. 50, no. 1, pp. 31-58.

9. Chartier, P., Murua, A. and Sanz-Serna, J. M., Higher-Order Averaging, Formal Series and Numerical Integration III: Error Bounds, Found. Comput. Math., 2015, vol. 15, no. 2, pp. 591-612.

10. Deprit, A. and Deprit-Bartholomé, A., Stability of the Triangular Lagrangian Points, The Astronomical Journal, 1967, vol. 72, no. 2, pp. 173-179.

11. dos Santos, F., Mansilla, J.E. and Vidal, C., Stability of Equilibrium Solutions of Autonomous and Periodic Hamiltonian Systems with $n$ Degrees of Freedom in the Case of Single Resonance, J. Dynam. Differential Equations, 2010, vol. 22, no. 4, pp. 805-821.

12. dos Santos, F. and Vidal, C., Stability of Equilibrium Solutions of Autonomous and Periodic Hamiltonian Systems in the Case of Multiple Resonances, J. Differential Equations, 2015, vol. 258, no. 11, pp. 38803901. 
13. Dumas, H.S., Meyer, K. R., Palacián, J.F. and Yanguas, P., Asymptotic Stability Estimates Near an Equilibrium Point, J. Differential Equations, 2017, vol. 263, no. 2, pp. 1125-1139.

14. Giorgilli, A., Delshams, A., Fontich, E., Galgani, L. and Simó, C., Effective Stability for a Hamiltonian System Near an Elliptic Equilibrium Point, with an Application to the Restricted Three Body Problem, J. Differential Equations, 1989, vol. 77, no. 1, pp. 167-198.

15. Han, Y., Li, Y. and Yi, Y., Invariant Tori in Hamiltonian Systems with High Order Proper Degeneracy, Ann. Henri Poincaré, 2010, vol. 10, no. 8, pp. 1419-1436.

16. Khazin, L. G., On the Stability of Hamiltonian Systems in the Presence of Resonances, J. Appl. Math. Mech., 1971, vol. 35, no. 3, pp. 384-391; see also: Prikl. Mat. Mekh., 1971, vol. 35, no. 3, pp. $423-431$.

17. Lerman, L. M. and Markova, A.P., On Stability at the Hamiltonian Hopf Bifurcation, Regul. Chaotic Dyn., 2009, vol. 14, no. 1, pp. 148-162.

18. Lochak, P., Canonical Perturbation Theory Via Simultaneous Approximation, Russian Math. Surveys, 1992, vol. 47, no. 6 , pp. 57-133.

19. Markeev, A.P., On the Stability of a Nonautonomous Hamiltonian System with Two Degrees of Freedom, J. Appl. Math. Mech., 1969, vol. 33, pp. 550-557; see also: Prikl. Mat. Mekh., 1969, vol. 33, no. 3, pp. 563569.

20. Markeev, A. P., Stability of the Triangular Lagrangian Solutions of the Restricted Three-Body Problem in the Three-Dimensional Circular Case, Soviet Astronomy, Astronomical Journal, 1972, vol. 15, no. 4, pp. 682-686; see also: Astronomicheskii Zhurnal, 1971, vol.48, no. 4, pp. 862-868.

21. Markeev, A.P., On the Stability Problem for the Lagrange Solutions of the Restricted Three-Body Problem, J. Appl. Math. Mech., 1973, vol.37, no.4, pp.713-717; see also: Prikl. Mat. Mekh., 1973, vol. 37, no. 4, pp. 753-757.

22. Markeev, A.P., Libration points in celestial mechanics and astrodynamics, Moscow: Moscow Izdatel'stvo Nauka, 1978 (Russian).

23. Meyer, K. R. and Offin, D., Introduction to Hamiltonian dynamical systems and the n-body problem, 3rd ed., New York: Springer-Verlag, 2017.

24. Meyer, K. R., Palacián, J.F. and Yanguas, P., Stability of a Hamiltonian System in a Limiting Case, Regul. Chaotic Dyn., 2012, vol. 17, no. 1, pp. 24-35.

25. Niven, I. M., Numbers: rational and irrational, New York: Random House, 1961.

26. Pöschel, J., On Nekhoroshev's Estimate at an Elliptic Equilibrium, Int. Math. Res. Not. IMRN, 1999, vol. 1999, no. 4, pp. 203-215.

27. Schirinzi, G. and Guzzo, M., Numerical Verification of the Steepness of Three and Four Degrees of Freedom Hamiltonian Systems, Regul. Chaotic Dyn., 2015, vol. 20, no. 1, pp. 1-18.

28. Sokol'skii, A. G., Proof of the Stability of Lagrange Solutions at a Critical Relation of Masses, Soviet Astronomy Letters, 1978, vol.4, no.2, pp.79-81; see also: Pis'ma v Astronomicheskii Zhurnal, 1978, vol. 4, no. 3, pp. 148-152.

29. Szebehely, V., Theory of orbits: the restricted problem of three bodies, New York and London: Academic Press, 1967. 